\title{
Dramatic response from neoadjuvant, spatially fractionated GRID radiotherapy (SFGRT) for large, high-grade extremity sarcoma
}

\author{
Adeel Kaiser • Majid M. Mohiuddin • \\ Gilchrist L. Jackson
}

Received: 6 August 2012 / Accepted: 20 August 2012 / Published online: 31 August 2012

(C) Springer-Verlag 2012

\section{Introduction}

Extremity sarcomas are managed surgically with the goal of limb preservation. In order to reduce local recurrence rates, radiation treatment has been used in either the preoperative or postoperative setting for select cases [1]. This combination has proven beneficial for large, high-grade, deeply invasive, or incompletely resected tumors [2]. Due to lower long-term complications rates, neoadjuvant treatment has become favored in cases where predicted postsurgical risks are low [3].

However, high-grade sarcomas rarely completely regress after preoperative radiation $[4,5]$. In addition, the median pathological treatment response for high-grade tumors is only $50 \%$, and patients with pathological necrosis rates less than $95 \%$ are 2.5 times more likely to develop a local recurrence $[5,6]$. Thus, very large $(\geq 8 \mathrm{~cm})$ sarcomas represent an even greater clinical challenge. This has prompted the addition of chemotherapy to preoperative radiation in the hopes of improving tumor response [6-8] and to evaluate effectiveness of the drugs. Although neoadjuvant chemoradiation shows greater tumor necrosis, it comes at a significant cost. One study showed a $90 \%$ risk of grades 3-4 hematologic toxicity with chemoradiation, and a multi-institutional phase II study reported increased hospitalizations and death $[7,8]$.

A. Kaiser $(\square) \cdot$ M. M. Mohiuddin

Northwest Radiation Oncology,

13700 Medical Complex Drive,

Houston, TX 77375, USA

e-mail: adeel.kaiser.md@gmail.com

G. L. Jackson

Kelsey-Seybold Clinic,

2727 West Holcombe Boulevard,

Houston, TX 77025, USA

\section{Methods}

We present an 82-year-old female with a right rapidly growing upper extremity sarcoma deemed medically unfit for systemic chemotherapy. Pathology results from biopsy demonstrated high-grade spindle cell sarcoma.

Neoadjuvant conventional external beam radiotherapy (cEBRT) followed by definitive surgery was planned. CT simulation with MRI imaging was used to contour the gross tumor volume (GTV), which measured as $527 \mathrm{~cm}^{3}$. The treated area included the GTV with $5-\mathrm{cm}$ proximal and distal margins. The lateral margin measured over $2.5 \mathrm{~cm}$, but the medial margin was as close as $0.7 \mathrm{~cm}$ in some areas to spare a strip of soft tissue and limit toxicity. Since the tumor was adjacent to the humerus, much of the bone was within the treatment field. An anterior-posterior, field-infield, beam arrangement was used with 6-MV photons (Fig. 1a-b). A dose of 50 Gy in once daily 2-Gy fractions was prescribed to the isocenter. However, setup verification films completed on the first day of radiation indicated that the tumor had grown beyond the field borders. Repeat CT simulation demonstrated a $20 \%$ increase in tumor volume to $631 \mathrm{~cm}^{3}$. The field borders were adjusted to reflect the increase, and the patient was able to start radiation the same day as originally planned.

After 10 Gy of cEBRT, the tumor volume had increased another $47 \%$ to $926 \mathrm{~cm}^{3}$, with finger-like tumor projections now breaking through the skin surface of the right upper arm. In the hopes of stemming further disease progression, the patient was emergently treated with spatially fractionated GRID radiotherapy (SFGRT) to the bulk of the tumor volume. A dose of $18 \mathrm{~Gy}$ in a single fraction was delivered to a depth of $2.8 \mathrm{~cm}$ (Dmax) with 15-MV photons. Therefore, the skin surface dose was approximately $5.3 \mathrm{~Gy}$, and the estimated dose at $1-\mathrm{cm}$ depth was $15 \mathrm{~Gy}$. The humerus 
Fig. 1 Neoadjuvant radiation treatment. a Right upper arm light field for conventional external beam radiation (cEBRT). b Digitally reconstructed radiograph showing field borders for right upper arm cEBRT with gross tumor volume (GTV) outlined in green. c Right upper arm light field for spatially fractionated GRID radiotherapy with tumor penetrating through the skin indicated by a blue arrow. d Digitally reconstructed radiograph showing field borders for right upper arm GRID field with GTV outlined in green (note: the medial aspect of the GTV is blocked by multi-leaf collimators with $1-\mathrm{cm}$ margin around the humerus)
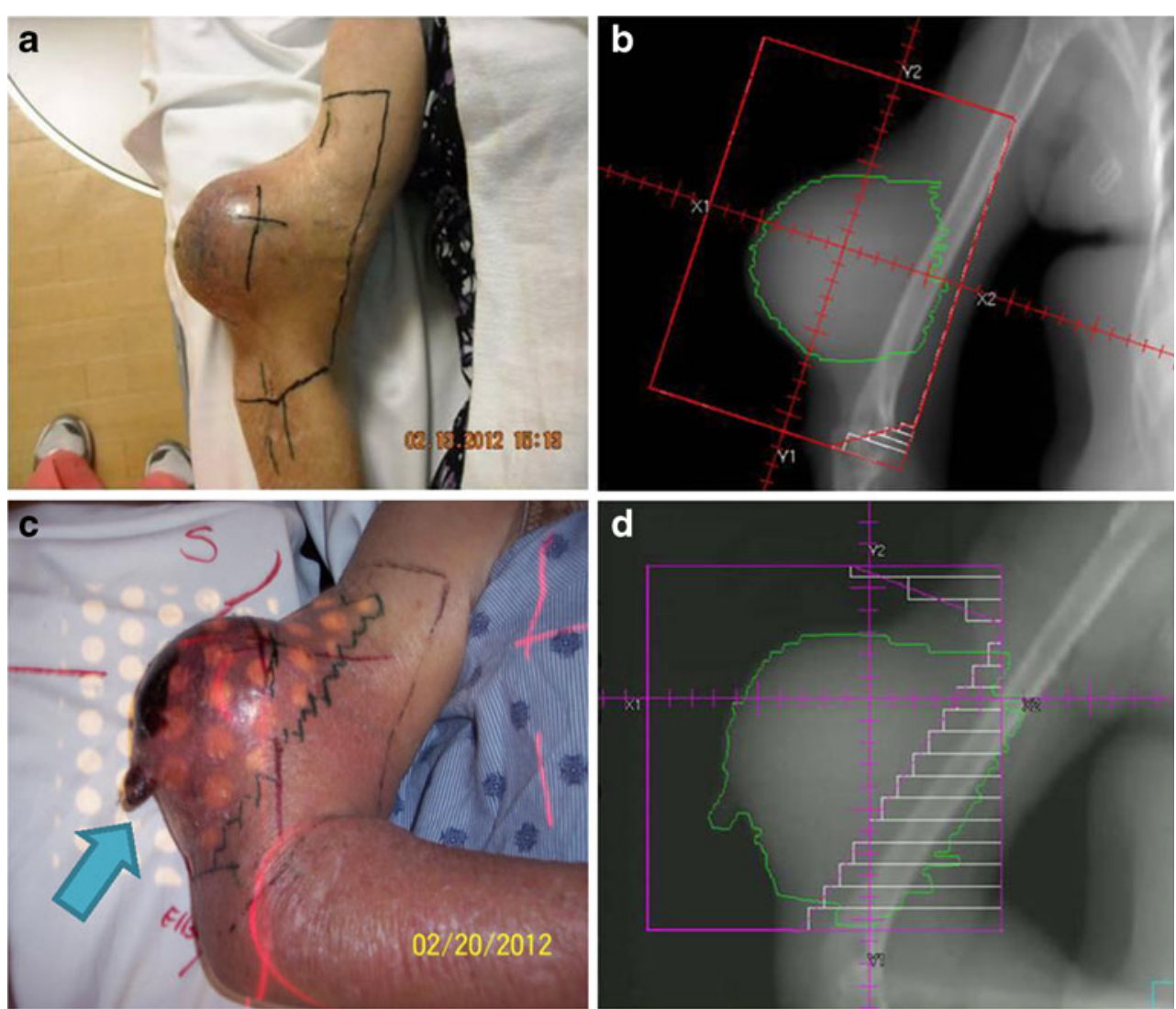

with 1-cm margin was shielded from the SFGRT field, and so the medial portion of the GTV was not treated with SFGRT (Fig. 1c-d). The GRID has an open-hole to closed-block surface-area ratio of 50:50, as previously described [9].

After SFGRT, the patient completed 11 more fractions of cEBRT for a total of 32 Gy by cEBRT and 18 Gy by SFGRT.

\section{Results}

Tumor growth was arrested within 10 days of SFGRT treatment. Throughout the remainder of cEBRT, and for several weeks thereafter, necrotic tumor debris slowly extruded through skin surface openings created by the tumor before SFGRT. Other than the skin defect created by the tumor itself, there were no residual skin toxicities prior to surgery (Fig. 2c). A radical resection of the ulcerated tumor with resection of the triceps muscle was performed 6.5 weeks after irradiation. She was reconstructed with an innervated right latissimus dorsi myocutaneous flap. There were no wound complications following surgery (Fig. 2f). Postoperative pathology demonstrated $65 \mathrm{~cm}^{3}$ of residual tumor with only $5-10 \%$ viable cells in a background of extensive fibrosis and necrosis. Surgical margins were clear by $\geq 1.2 \mathrm{~cm}$ in all directions. Based on a pre-treatment tumor volume of $631 \mathrm{~cm}^{3}$, our patient experienced tumor regression and necrosis rates of 90 and $99 \%$, respectively (Fig. 2c-e).

\section{Discussion}

We report a case of a very large spindle cell sarcoma that rapidly progressed even during the first week of cEBRT. In order to arrest growth and avoid limb amputation, we used GRID to deliver a high dose of one-time radiation, followed by more cEBRT.

The treatment response of $90 \%$ seen in our patient is dramatic in comparison to studies showing a meager 0 $0.5 \%$ radiological regression rate for high-grade tumors after cEBRT of 50 Gy $[4,5]$. More importantly, the patient achieved negative surgical margins even though the medial portion of the tumor surrounding the humerus was directly shielded during SFGRT. This area regressed though it received only 32 Gy from cEBRT, well below the standard preoperative dose of $50 \mathrm{~Gy}$.

Our patient's tumor response may be attributed to a bystander effect from SFGRT, which indirectly killed tumor cells adjacent to the treated area. SFGRT functions like virtual brachytherapy where stereotactic, high-dose beamlets of external beam radiation traverse the tumor in a dosimetric pattern akin to interstitial catheters [9]. These 

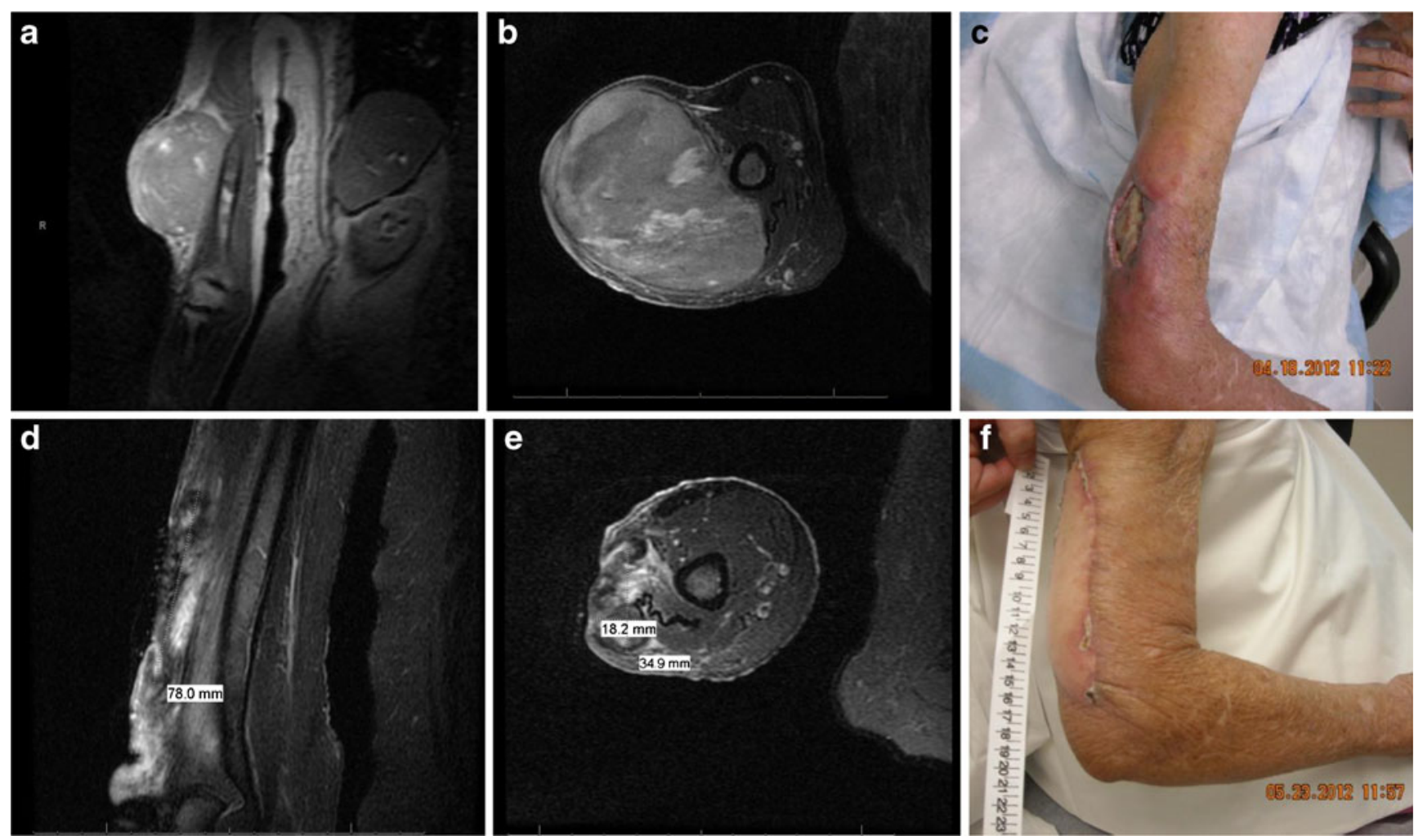

Fig. 2 Clinical and radiographic tumor response to neoadjuvant radiation. a Coronal MRI of right upper arm prior to start of radiation treatment. b Axial MRI of right upper arm prior to start of radiation treatment. c Right upper arm before surgery and 6 weeks after completion of radiation. $\mathbf{d}$ Coronoal MRI of right upper arm 6 weeks after completion of radiation. e Axial MRI of right upper arm 6 weeks after completion of radiation. $\mathbf{f}$ Right upper arm 4 weeks after radical resection and innervated right latissimus dorsi myocutaneous flap placement high-dose islands cause intense direct cell kill and indirect bystander death in adjacent or blocked areas of tumor by endemically secreted cytokines like TNF-alpha [10]. The GRID block technique allows for coverage of a larger field size of tumor than can be tolerated through an open portal [9]. For normal tissues, like the skin, blocked areas provide sparing and serve as repositories for normal cell migration and healing. Bringing in a healthy, well-vascularized myocutaneous flap facilitated primary healing of the radical resection site.

Our results suggest that a combination of SFGRT and cEBRT may be a superior neoadjuvant treatment approach than cEBRT with or without chemotherapy. With a $99 \%$ necrosis rate of this high-grade sarcoma, the addition of SFGRT decreases the need for chemotherapy and limits toxicity during neoadjuvant therapy. Furthermore, since only 32 Gy was given to the tumor near the humerus, there is now room for additional radiation postoperatively in the case of a positive margin. These advantages illustrate why SFGRT can be safely integrated into neoadjuvant strategies for large extremity sarcomas.

Conflict of interest The authors declare that they have no conflict of interest.

\section{References}

1. Rosenberg SA, Tepper J, Glatstein E et al (1982) The treatment of soft-tissue sarcomas of the extremities: prospective randomized evaluations of (1) limb-sparing surgery plus radiation therapy compared with amputation and (2) the role of adjuvant chemotherapy. Ann Surg 196:305-315

2. Zagars GK, Ballo MT, Pisters PW, Pollock RE, Patel SR, Benjamin RS, Evans HL (2003) Prognostic factors for patients with localized soft-tissue sarcoma treated with conservation surgery and radiation therapy: an analysis of 1225 patients. Cancer 97:2530-2543

3. O'Sullivan B, Davis AM, Turcotte R et al (2002) Preoperative versus postoperative radiotherapy in soft-tissue sarcoma of the limbs: a randomised trial. Lancet 359:2235-2241

4. Canter RJ, Martinez SR, Tamurian RM, Wilton M, Li CS, Ryu J, Mak W, Monsky WL, Borys D (2010) Radiographic and histologic response to neoadjuvant radiotherapy in patients with soft tissue sarcoma. Ann Surg Oncol 17:2578-2584

5. Roberge D, Skamene T, Nahal A, Turcotte RE, Powell T, Freeman C (2010) Radiological and pathological response following preoperative radiotherapy for soft-tissue sarcoma. Radiother Oncol 97:404-407

6. Eilber FC, Rosen G, Eckardt J, Forscher C, Nelson SD, Selch M, Dorey F, Eilber FR (2001) Treatment-induced pathologic necrosis: a predictor of local recurrence and survival in patients receiving neoadjuvant therapy for high-grade extremity soft tissue sarcomas. J Clin Oncol 19:3203-3209 
7. MacDermed DM, Miller LL, Peabody TD, Simon MA, Luu HH, Haydon RC, Montag AG, Undevia SD, Connell PP (2010) Primary tumor necrosis predicts distant control in locally advanced softtissue sarcomas after preoperative concurrent chemoradiotherapy. Int J Radiat Oncol Biol Phys 76:1147-1153

8. Kraybill WG, Harris J, Spiro IJ et al (2006) Phase II study of neoadjuvant chemotherapy and radiation therapy in the management of high-risk, high-grade, soft tissue sarcomas of the extremities and body wall: radiation therapy oncology group trial 9514. J Clin Oncol 24:619-625
9. Mohiuddin M, Fujita M, Regine WF, Megooni AS, Ibbott GS, Ahmed MM (1999) High-dose spatially-fractionated radiation (GRID): a new paradigm in the management of advanced cancers. Int J Radiat Oncol Biol Phys 45:721-727

10. Sathishkumar S, Dey S, Meigooni AS, Regine WF, Kudrimoti MS, Ahmed MM, Mohiuddin M (2002) The impact of TNF-alpha induction on therapeutic efficacy following high dose spatially fractionated (GRID) radiation. Technol Cancer Res Treat 1:141147 